\title{
A modified fuzzy C-means method for segmenting MR images using non-local information
}

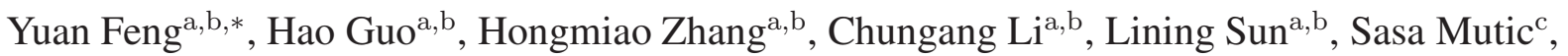 \\ Songbai $\mathrm{Ji}^{\mathrm{d}}$ and Yanle $\mathrm{Hu}^{\mathrm{c}, \mathrm{e}}$ \\ ${ }^{a}$ School of Mechanical and Electronic Engineering, Soochow University, Suzhou, Jiangsu, China \\ ${ }^{\mathrm{b}}$ Robotics and Microsystems Center, Soochow University, Suzhou, Jiangsu, China \\ ${ }^{\mathrm{c}}$ Department of Radiation Oncology, Washington University, St. Louis, MO, USA \\ ${ }^{\mathrm{d}}$ Thayer School of Engineering, Dartmouth College, Hanover, NH, USA \\ e Department of Radiation Oncology, Mayo Clinic, Phoenix, AZ, USA
}

\begin{abstract}
.
BACKGROUND: In recent years, MR images have been increasingly used in therapeutic applications such as image-guided radiotherapy (IGRT). However, images with low contrast values and noises present challenges for image segmentation.

OBJECTIVE: The objective of this study is to develop a robust method based on fuzzy C-means (FCM) method which can segment MR images polluted with Gaussian noise.

METHODS: A modified FCM algorithm accommodating non-local pixel information via Hausdorff distance was developed for segmenting MR images. The membership and objective functions were modified accordingly. Segmentations with different weights of the Hausdorff distance were compared.

RESULTS: Segmentation tests using synthetic and MR images showed that the proposed algorithm was better at resolving boundaries and more robust to Gaussian noise. By segmenting a sample MR image of a tumor, we further showed the capability of the method in capturing the centroid of the target region.

CONCLUSIONS: The modified FCM algorithm with neighboring information can be used to segment blurry images with potential applications in segmenting motion MR images in image-guided radiotherapy (IGRT).
\end{abstract}

Keywords: Segmentation, Hausdorff distance, fuzzy C-means, motion images, MR images

\section{Introduction}

Image segmentation plays an important role in medical image analysis [1-3]. Among the typical medical image modalities, Magnetic Resonance (MR) images are particularly attractive due to their superior soft tissue contrast, non-radiation effect, varieties of functional imaging methods, and the capability of motion imaging. Besides conventional diagnostic applications, MR images have been increasingly used in therapeutic applications such as tracking certain region of interest (ROI) in image-guided radiotherapy (IGRT) [4-6]. In recent development of IGRT, segmentation of MR images in magnetic resonance image-guided radiation treatment (MR-IGRT) $[6,7]$ is getting more attention.

\footnotetext{
${ }^{*}$ Corresponding author: Yuan Feng, School of Mechanical and Electronic Engineering, Soochow University, Suzhou, Jiangsu, China. E-mail: fengyuan@ suda.edu.cn.
}

0928-7329/16/\$35.00 @ 2016 - IOS Press and the authors. All rights reserved This article is published online with Open Access and distributed under the terms of the Creative Commons Attribution NonCommercial License. 
Many pattern recognition methods have been applied in segmenting MR images [8,9]. Fuzzy Cmeans (FCM) clustering, a typical unsupervised clustering method, has shown to have a robust performance [10]. It has been widely used in medical image segmentation [11-13]. Based on FCM, the purpose of this study is to address a general Gaussian-type noise existing in many MR images, and to explore its potential in segmenting motion MR images. The improvement of FCM has been an active research field for years. For example, Pham (2001) proposed a spatial penalty term for the membership function [14], and showed the modified algorithm is more robust to noise and artifacts. Kannan et al. used a kernel with defined tangent to modify the membership function [15]. Zhang et al. (2012) adopted a genetic algorithm to achieve the minimization of the objective function, and demonstrated an improved performance of the algorithm to find the global minimum of the objective function [16]. Noreen et al. (2011) applied a wavelet transform to images as a pre-processing before FCM segmentation so that the algorithm is more robustness to noise [17]. Keller et al. (2011) used an adaptive method to determine the cluster group numbers so that optimal clusters were acquired based on tissue properties [18]. Probability theory was also introduced to include membership and probability simultaneously [19]. Recently, a relational FCM (RFCM) method was also introduced [20]. These modifications of the FCM algorithm improve the algorithm from different perspectives with changes to membership function, objective function, or the cluster group selection. However, the measure between pixels for clustering is based on a standard measure in the Euclid space, with only local pixel information.

The measure in Euclid space between single pixels limits the capability of FCM in segmenting images with noise. To improve the performance of FCM, Caldairou et al. (2011) used a non-local FCM method to segment brain MR images [21]. New forms of membership functions and objective functions including neighbor information were also proposed to increase the performance of FCM [22-26]. In these studies, membership functions were modified to have a weight function to include non-local pixel information from adjacent pixels, and the objective function was modified to include a non-local regularization term [21]. Cao et al. (2012) also utilized the non-local information by applying a convolution kernel to a region of pixels in a modified FCM objective function [27]. Among these methods, a separate functional has to be introduced to include the non-local information for the membership clustering.

Recently, researchers have been exploring different ways to measure distance in various imageprocessing algorithms. For example, Riemann scalar measure has been applied to diffusion tensor imaging [28]. An early work by Huttenlocher et al. found that Hausdorff distance is especially useful in dealing with images with small perturbation [29]. In this work, we employed the Hausdorff distance in FCM so that non-local information could be used by a single distance measurement. Synthetic images as well as conventional and motion MR images were used to evaluate the modified FCM method. Segmentation results were compared with those from the conventional FCM method.

\section{Materials and methods}

\subsection{FCM algorithm}

The FCM algorithm allocates each pixel to each pre-defined category using a fuzzy membership function [30]. An iterative optimization process is used to minimize the objective function:

$$
J=\sum_{i=1}^{n} \sum_{k=1}^{c} u_{i k}^{m}\left\|x_{i}-v_{k}\right\|^{2}
$$

where $x_{i}(i=1, \ldots, n)$ is the pixel value to be allocated into a cluster group, $v_{k}(k=1, \ldots, c)$ is the center value of each cluster, $u_{i k}$ is the membership function allowing each pixel to belong to all 
the cluster groups to a certain extent, and $m$ is the weighting exponent describing the fuzziness of classification [10]. The membership function has to satisfy

$$
\sum_{k=1}^{c} u_{i k}=1,0 \leqslant u_{i k} \leqslant 1, \forall i, k .
$$

The membership functions and the cluster centers are updated at each iteration by

$$
\begin{aligned}
& u_{i k}=\frac{\left\|x_{i}-v_{k}\right\|^{\frac{-2}{m-1}}}{\sum_{l=1}^{c}\left\|x_{i}-v_{l}\right\|^{\frac{-2}{m-1}}}, \\
& v_{k}=\frac{\sum_{i=1}^{n} u_{i k}^{m} x_{i}}{\sum_{i=1}^{n} u_{i k}^{m}} .
\end{aligned}
$$

After initialization of the cluster center, the membership function and center are updated at each iteration until the change of the objective function is below a preset threshold.

\subsection{Modified FCM algorithm}

In order to utilize the non-local information to increase the robustness of the algorithm, similar to Ahmed et al. (2002), Kang et al. (2009) used an adaptive weighted averaging image to compute the membership function. Studies have also shown that the use of different distance measure besides Euclid norm such as Mahanolobis norm can influence the clustering results $[9,10]$. In this study, we investigate the use of Hasudorff distance to accommodate the non-local information. Let $A$ and $B$ be two nonempty subsets of Euclidean metric space $\mathbf{R}$ with the conventional metric defined by an absolute value. The Hausdorff distance $d_{H}$ between the two sets is

$$
d_{H}(A, B)=\max \left\{\sup _{x \in A} \inf _{y \in B}|y-x|, \sup _{y \in B} \inf _{x \in A}|y-x|\right\} .
$$

Based on the definition of Hausdorff distance, we define the distance measure between two pixels $x$ and $y$ to be

$$
\|x-y\|_{H}=d_{H}\left(\Omega_{x}, \Omega_{y}\right)
$$

where $\Omega_{x}\left(\Omega_{y}\right)$ is a pixel subset containing $x(y)$ and its adjacent 8-connected pixels. Thus, the number of elements of the subsets $\left|\Omega_{x}\right|$ and $\left|\Omega_{y}\right|$ is 9 . In cases when the pixel is at the boundary of an image (on sides or at corners of an image), the 8-connected adjacent subsets is truncated by the edge of the image to preserve the neighbor information. It is apparent that $|\Omega|$ is 6 or 4 when the pixel is on the sides of the image or at the corners of the image, respectively.

We modify the objective function using the Hausdorff distance defined:

$$
J=\sum_{i=1}^{n} \sum_{k=1}^{c} u_{i k}^{m}\left\|x_{i}-v_{k}\right\|^{2}+\alpha \sum_{i=1}^{n} \sum_{k=1}^{c} u_{i k}^{m}\left\|x_{i}-v_{k}\right\|_{H}^{2},
$$

where $\alpha$ is the ratio determining the contribution of non-local information from the neighbor pixels. Correspondingly, the membership function is updated by

$$
u_{i k}=\frac{\left(\left\|x_{i}-v_{k}\right\|^{2}+\alpha\left\|x_{i}-v_{k}\right\|_{H}^{2}\right)^{\frac{-1}{m-1}}}{\sum_{l=1}^{c}\left(\left\|x_{i}-v_{l}\right\|^{2}+\alpha\left\|x_{i}-v_{l}\right\|_{H}^{2}\right)^{\frac{-1}{m-1}}},
$$

where $m$ is taken as 2 . The cluster center is updated as in Eq. (4). When $\alpha=0$, it is easy to verify that the modified the FCM is the same as the standard FCM algorithm. To initialize the iteration process to find the cluster center, initial values of the center are chosen to span the image intensity values equally. The iteration ends when the change of $J$ is less than $10^{-6}$. 
(a)

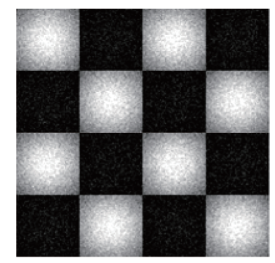

(b)

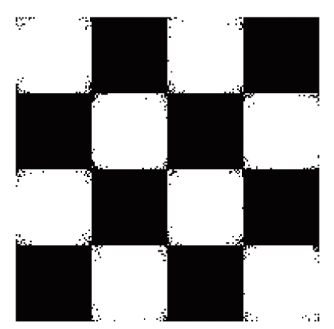

(d)

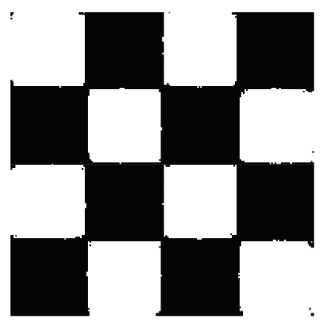

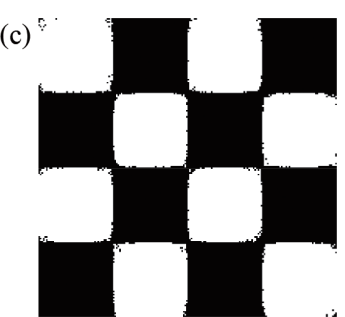

(e)

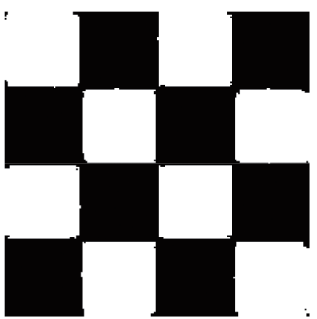

Fig. 1. (a) A synthetic checkerboard pattern with modulated intensity and Gaussian noise added. The Gaussian noise has a zero mean and a standard deviation of 5\%. (b) Segmentation by FCM. (c, d, e) Segmentation results from the modified FCM by setting $\alpha=0.5,5$, and 50 , respectively.

\subsection{Synthetic images}

The Hausdorff distance implemented in the modified FCM is able to include non-local information for the iterative clustering process. To demonstrate the advantages of the modified FCM in resolving blurred boundaries and show its robustness in segmentation, we created synthetic images to test the method.

A gray scale checkerboard pattern is generated with its intensity modulated at each grid by a $2 \mathrm{D}$ Gaussian function. The synthetic pattern has a resolution of $192 \times 192$ pixels. The modulation Gaussian function applied to each grid has a standard deviation of 12 pixels. To test the performance of the modified FCM in segmenting images with noise, Gaussian noise is added to the checkerboard pattern with a zero mean and a standard deviation of $0.5 \%$ (Fig. 1(a)). In segmenting the synthetic checkerboard pattern, two cluster groups are used for both FCM and the modified FCM. Initial cluster centers are taken as 0 and 1 , spanning the two ends of the gray scale image. To investigate the effect of the inclusion of non-local information, we compare the segmentation results from the modified FCM (with $\alpha$ equals to $0.5,5$, and 50) with those from FCM.

\subsection{Conventional anatomical MR images}

A T1-weighted MR image of human brain (Fig. 2(a)) with a resolution of $512 \times 512$ pixels was selected from an online image database (Nagoya Hospital, http://podcast-nagoya.net/XRay/). We used the T1-weighted image to compare the performance of FCM and the modified FCM in segmenting white matter (WM), gray matter (GM), and cerebral spinal fluid (CSF). Three cluster groups are used for segmentation, representing the ROI of GM, WM, and CSF. Initial cluster centers are taken as equally spaced pixel values from the image histogram.

\subsection{Motion MR images from an MRgRT system}

To investigate the potential implementation of the modified FCM in segmenting motion MR images, we applied the modified algorithm to a sample motion image acquired from a commercial MR-IGRT 

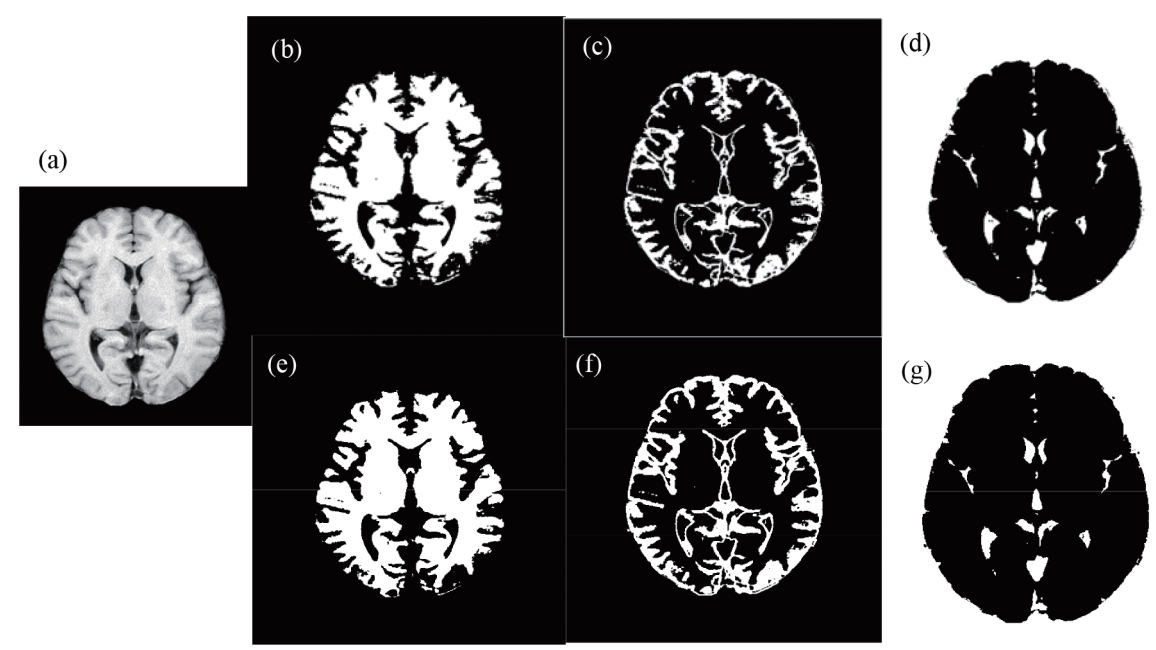

Fig. 2. (a) A T1-weighted anatomy image of normal human brain. Segmentation of WM (b, e), GM (c, f), and CSF (d, g) by FCM and the modified FCM, respectively. The value of $\alpha$ for the modified FCM is taken as 50.

system [6]. A field of view (FOV) of $351 \times 450 \mathrm{~mm}^{2}$ with a pixel size of $3.5 \times 3.5 \mathrm{~mm}^{2}$ is selected to show a tumor in liver (Fig. 3(a)). Histogram equalization is applied as a pre-processing step in segmentation of the liver tumor, since the image contrast is low. Three cluster groups were used based on a test-run selecting the pixel groups containing the ROI.

\section{Results}

\subsection{Segmentation of synthetic images}

Because of the presence of Gaussian noise, segmentation result from FCM has scattered dots around the corners (Fig. 1(b)). For segmentation with the modified FCM, the segmentation results gradually improve with an increase of $\alpha$ (Figs 1(c)-1(e)). When $\alpha=0.5$, noisy spots can still be observed at the edges and corners of each grid. When $\alpha=5$ (Fig. 1(d)), fewer noisy spots are observed. The performance is improved further when $\alpha=50$ (Fig. 1(e)).

\subsection{Segmentation of conventional diagnostic MR images}

Figure 2 shows the segmentation results of the brain anatomical image. Compared with FCM (Figs 2(b)-2(d)), the modified FCM method (Figs 2(e)-2(g)) is able to segment the ROI with less noise points. Also, the modified FCM can delineate the boundary of the ROI better than FCM.

To demonstrate the effect of inclusion of non-local information, we magnify the WM and GM segmentations from different $\alpha$ values in the frontal lobe section (Figs 4(c)-4(h)). We also observe that an increasing value of $\alpha$ generates a better segmentation. It is apparent to see that the WM and GM boundaries are smoothly separated from the boundary tissues with the modified FCM. The FCM also demonstrates to have more missing pixel points inside of the segmented region. 


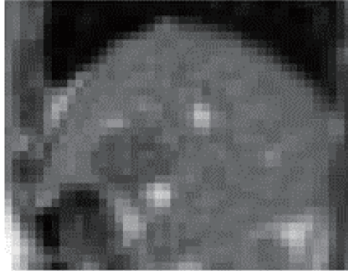

(a)

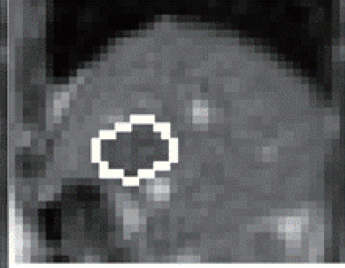

(b)

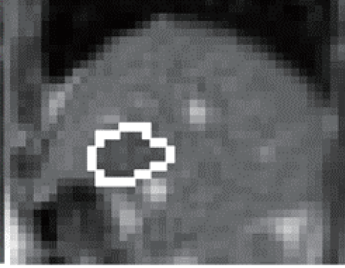

(c)

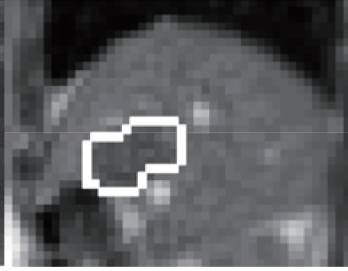

(d)

Fig. 3. (a) A sample motion MR image containing a liver tumor. (b) Manual segmentation of the liver tumor. Segmentation of the tumor from (c) FCM, and (d) the modified FCM. The white line is the boundary of the segmented tumor region. The value of $\alpha$ for the modified FCM is taken as 50 .

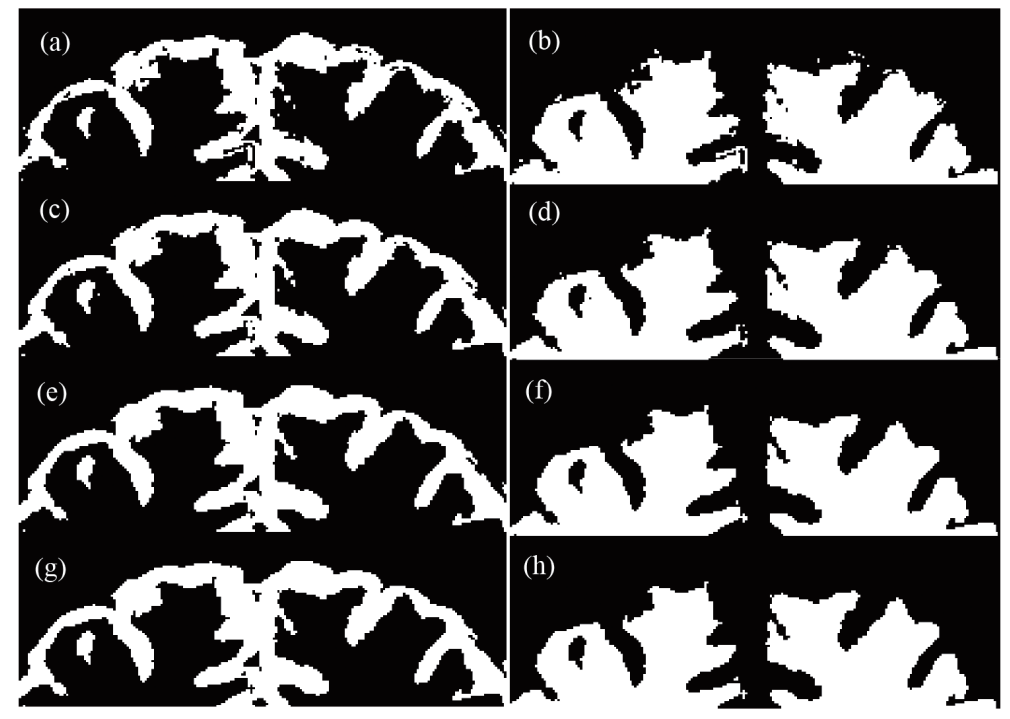

Fig. 4. Comparison of the segmentation results of a section of the frontal lobe from Fig. 2 of GM (a, c, e, g) and WM (b, d, f, h) by FCM (a, b) and the modified FCM with $\alpha=0.5$ (c, d), 5 (e, f), 50 (g, h), respectively.

\subsection{Segmentation of motion MR images from an MR-IGRT system}

We take the manually delineated tumor ROI as ground truth (Fig. 3(b)). The modified FCM segmented out a region larger (Fig. 3(d)) than that of FCM (Fig. 3(e)). Table 1 shows the Jaccard index from FCM and the modified FCM. To achieve a conformal radiotherapy, accurate tracking of the centroid position of the target region in IGRT is important. Thus, we calculated the geometric centroid of the segmented region for comparison. The calculated the position error is

$$
(\Delta x, \Delta y)=\left(\left|x_{\text {seg }}-x_{0}\right|,\left|y_{\text {seg }}-y_{0}\right|\right),
$$

where $\left(x_{0}, y_{0}\right)$ is the geometric centroid position of the ground truth region, and $\left(x_{\text {seg }}, y_{\text {seg }}\right)$ is the geometric centroid position of the segmented ROI. A comparison of the two methods shows that the modified FCM generates a smaller position error than FCM (Table 1). 
Table 1

Jaccard index and the position error of the centroid of geometric ROI from FCM and the modified FCM method. The error is presented by an absolute difference $(x, y)$ of the coordinates between the centroid position from the segmented and the manually delineated ROI. Unites are in $\mathrm{mm}$

\begin{tabular}{lcc}
\hline & FCM & The modified FCM \\
\hline Jaccard & 0.81 & 0.68 \\
$(x, y)$ & $(1.58,2.22)$ & $(0.64,1.38)$ \\
\hline
\end{tabular}

\section{Discussion}

We modified FCM algorithm by accommodating non-local information via Hausdorff distance in the iterative clustering process, and showed the advantages of the algorithm in segmenting MR images. By comparing the segmentation results of the synthetic image and the conventional anatomical MR image, we are able to show that the modified FCM is more robust to Gaussian noise. The potential application of the modified FCM in segmenting motion MR images is also demonstrated by segmenting a sample image of liver tumor.

Although a soft membership function is used in FCM, the method is limited in distinguishing the neighbor region once Gaussian noise is presented. One of the advantages of the modified FCM is the inclusion of non-local information at each pixel location. This is achieved by using Hausdorff distance to measure pixels based on its surrounding neighboring region [3]. Similar non-local FCM methods include a Euclid distance by two "patch" pixels proposed by Caldairou et al. [21]. Other modification of FCM by adopting different distance measure such as a hyper function tangent showed to improve the convergence rate. However, information used for clustering is still confined to local pixels. The intrinsic properties of the Hausdorff measure can extract the maximum similarity information of the neighbor pixels, thus shows to have a robust performance in the image exposed to Gaussian noise.

Although the Jaccard indices of FCM are higher than that of the modified FCM in segmenting the liver tumor, the centroid position of the target ROI was tracked better by the modified FCM. This feature of the modified FCM is desirable in MR-IGRT, since tracking the target volume in motion images is important in achieving conformal radiotherapy. Also, the modified FCM can generate a better-resolved boundary, thus reducing the aftermath morphological processing procedures for the target ROI selection.

However, the processing time of segmentation is elongated due to the inclusion of non-local information in the modified FCM. Also, optimal utilization of the non-local information and the choice of cluster groups in segmentation motion images still need to be improved.

\section{Conclusion}

A modified FCM method is used to segment MR images. Segmentation of synthetic patterns and conventional anatomic MR images show the modified FCM is more robust to Gaussian noise and has better performances in resolving interface boundaries. Segmentation tests of motion MR images from a commercial MR-IGRT system also show the potential of the modified FCM in tracking the geometric centroid of the target region. Future studies include an adaptive method to utilize the non-local information and application of the modified FCM to segment more motion images from the MR-IGRT system.

\section{Acknowledgments}

Grant BK20140356 (Feng), BK20130325 (Guo), and BK2012215 (Li) from Jiangsu Province, grant 61503267 (Feng) from NSFC, and Grant ICT1532 (Zhang) from Zhejiang University are acknowledged. 


\section{References}

[1] C. Petitjean and J.-N. Dacher, A review of segmentation methods in short axis cardiac MR images. Medical Image Analysis 15, 169 (2011).

[2] B.N. Li, C.K. Chui, S. Chang and S.H. Ong, Integrating spatial fuzzy clustering with level set methods for automated medical image segmentation. Computers in Biology and Medicine 41, 1 (2011).

[3] J. Egger, T. Kapur, A. Fedorov, S. Pieper, J.V. Miller, H. Veeraraghavan, B. Freisleben, A.J. Golby, C. Nimsky and R. Kikinis, GBM volumetry using the 3D Slicer medical image computing platform. Scientific Reports 3, 1364 (2013).

[4] Y. Hu, S.D. Caruthers, D.A. Low, P.J. Parikh and S. Mutic, Respiratory Amplitude Guided 4-Dimensional Magnetic Resonance Imaging. Int J Radiat Oncol 86, 198 (2013).

[5] J.J.W. Lagendijk, B.W. Raaymakers, C.A.T. Van den Berg, M.A. Moerland, M.E. Philippens and M. van Vulpen, MR guidance in radiotherapy. Phys Med Biol 59, R349 (2014).

[6] S. Mutic, P.J. Parikh, J.D. Bradley, D.E. Hallahan, Y. Hu, R. Kashani, I. Kawrakow, H. Li, J.M. Michalski, J.R. Olsen, C.G. Robinson, V. Rodriguez, L. Santanam, K. Tanderup, J. Victoria, H.O. Wooten, D. Yang, I. Zoberi, O.L. Green and J.F. Dempsey, The Dawn of a New Era: First Ever MR-IGRT Treatments - Initial Experiences and Future Implications. Int J Radiat Oncol 90, S94 (2014).

[7] D. Thomson, C. Boylan, T. Liptrot, A. Aitkenhead, L. Lee, B. Yap, A. Sykes, C. Rowbottom and N. Slevin, Evaluation of an automatic segmentation algorithm for definition of head and neck organs at risk. Radiat. Oncol. 9, 12 (2014).

[8] J.C. Bezdek, L.O. Hall and L.P. Clarke, Review of MR Image Segmentation Techniques Using Pattern-Recognition. Med Phys 20, 1033 (1993).

[9] J.C. Bezdek, Pattern Recognition with Fuzzy Objective Function Algorithms, Kluwer Academic Publishers (1981).

[10] J.C. Bezdek, R. Ehrlich and W. Full, FCM: The fuzzy C-means clustering algorithm. Computers \& Geosciences 10, 191 (1984).

[11] Y. Feng, P.V. Bayly, J. Huang, C. Robinson, D. Du, S. Mutic, J.S. Shimony, E.C. Leuthardt, R.J. Okamoto and Y. Hu, A simulation study to investigate the potential of using Magnetic Resonance Elastography (MRE) to differentiate recurrent tumor and radiation necrosis. Medical Physics 40, 540 (2013).

[12] X.Y. Wang and J.A. Bu, A fast and robust image segmentation using FCM with spatial information. Digit Signal Process 20,1173 (2010).

[13] A.K. Jain, M.N. Murty and P.J. Flynn, Data clustering: a review. ACM Computing Surveys 31, 264 (1999).

[14] D.L. Pham, Spatial models for fuzzy clustering. Comput Vis Image Und 84, 285 (2001).

[15] S.R. Kannan, S. Ramathilagam, R. Devi and A. Sathya, Robust kernel FCM in segmentation of breast medical images. Expert Systems with Applications 38, 4382 (2011).

[16] C. Zhang, P. Wang and C. Liu, Green Communications and Networks, edited by Y. Yang and M. Ma, Springer Netherlands (2012), pp. 219-226.

[17] N. Noreen, K. Hayat and S.A. Madani, MRI segmentation through wavelets and fuzzy C-means. World Applied Sciences Journal 13, 34 (2011).

[18] B. Keller, D. Nathan, Y. Wang, Y. Zheng, J. Gee, E. Conant and D. Kontos, Adaptive Multi-cluster Fuzzy C-Means Segmentation of Breast Parenchymal Tissue in Digital Mammography. Medical Image Computing and Computer-Assisted Intervention-MICCAI 2011, edited by G. Fichtinger, A. Martel and T. Peters, Springer Berlin Heidelberg (2011), pp. $562-569$.

[19] N.R. Pal, K. Pal, J.M. Keller and J.C. Bezdek, A possibilistic fuzzy C-means clustering algorithm. IEEE Transactions on Fuzzy Systems 13, 517 (2005).

[20] M.A. Khalilia, J. Bezdek, M. Popescu and J.M. Keller, Improvements to the relational fuzzy C-means clustering algorithm. Pattern Recogn 47, 3920 (2014).

[21] B. Caldairou, N. Passat, P.A. Habas, C. Studholme and F. Rousseau, A non-local fuzzy segmentation method: Application to brain MRI. Pattern Recogn 44, 1916 (2011).

[22] J.Y. Kang, L.Q. Min, Q.X. Luan, X. Li and J.Z. Liu, Novel modified fuzzy C-means algorithm with applications. Digit Signal Process 19, 309 (2009).

[23] Z. Yang, F.L. Chung and S.T. Wang, Robust fuzzy clustering-based image segmentation. Appl Soft Comput 9, 80 (2009).

[24] W.L. Cai, S.C. Chen and D.Q. Zhang, Fast and robust fuzzy C-means clustering algorithms incorporating local information for image segmentation. Pattern Recogn 40, 825 (2007).

[25] Y. Xia, D.G. Feng, T.J. Wang, R.C. Zhao and Y.N. Zhang, Image segmentation by clustering of spatial patterns. Pattern Recogn Lett 28, 1548 (2007).

[26] M.N. Ahmed, S.M. Yamany, N. Mohamed, A.A. Farag and T. Moriarty, A modified fuzzy C-means algorithm for bias field estimation and segmentation of MRI data. IEEE T Med Imaging 21, 193 (2002).

[27] H. Cao, H. Deng and Y. Wang, Segmentation of M-FISH images for improved classification of chromosomes with an adaptive fuzzy C-means clustering algorithm. IEEE Transactions on Fuzzy Systems 20, 1 (2012). 
[28] L. Astola, A. Fuster and L. Florack, A Riemannian scalar measure for diffusion tensor images. Pattern Recogn 44, 1885 (2011).

[29] D.P. Huttenlocher, G.A. Klanderman and W.J. Rucklidge, Comparing images using the Hausdorff distance. IEEE Transactions on Pattern Analysis and Machine Intelligence 15, 850 (1993).

[30] T.A. Runkler and J.C. Bezdek, Alternating cluster estimation: A new tool for clustering and function approximation. IEEE Transactions on Fuzzy Systems 7, 377 (1999). 